\title{
INTEGRAÇÃO ENERGÉTICA DA ETAPA DE EXTRAÇÃO DE ÓLEO DE SOJA UTILIZANDO ANÁLISE PINCH
}

\author{
Aparecido Nivaldo Módenes ${ }^{l}$ \\ Carlos Coutinho Fernandes Jr. ${ }^{1}$ \\ Guilherme Bazarin ${ }^{1}$ \\ Fernando Rodolfo Espinoza-Quiñones ${ }^{1}$ \\ Carlos Eduardo Borba ${ }^{1}$
}

\begin{abstract}
Resumo: Neste trabalho, foi realizado um estudo de caso de integração energética na etapa da extração de uma fábrica de óleo de soja operando com produção média de 15.000 ton/mês. Após uma análise nas correntes da etapa de extração, baseando-se no potencial de troca térmica, foram selecionadas quatro correntes. A primeira corrente quente (Q1), consiste de óleo bruto, com temperaturas de entrada $110^{\circ} \mathrm{C}$ e saída $80^{\circ} \mathrm{C}$. A segunda corrente quente (Q2), consiste de água que sai de um aquecedor à $90^{\circ} \mathrm{C}$ e necessita ser resfriada até $55^{\circ} \mathrm{C}$. A terceira corrente (F1) consiste de água que sai do decantador e necessita ser aquecida de $40^{\circ} \mathrm{C}$ até $90^{\circ} \mathrm{C}$. A quarta corrente $(\mathrm{F} 2)$ consiste em uma mistura de $70 \%$ óleo e $30 \%$ hexano com temperaturas de entrada $60^{\circ} \mathrm{C}$ e saída $90^{\circ} \mathrm{C}$. A metodologia adotada para a síntese da rede de trocadores de calor foi a Análise Pinch, devido à facilidade de aplicação e interação com o usuário. Após a integração foi feita uma análise dos custos e verificou-se uma economia de consumo de vapor de 79,6\% (5,3\% do consumo global da planta). No custo total anual há uma redução $77,5 \%$, após a síntese da rede. A taxa de retorno para o investimento proposto é de apenas 3 meses.
\end{abstract}

Palavras-chave: Análise Pinch, Integração Energética, Máxima Recuperação de Energia.

Abstract: In this paper, a case study of energy integration in extraction phase in a soybean oil factory was performed. Based on the thermal potential change, two hot and two cold currents were selected from a set of streams of soybean oil extraction phases. A first hot current (Q1) transports crude oil with 110 and $80^{\circ} \mathrm{C}$ as input and output temperatures, respectively, while the second one (Q2) transports water that comes out from a boiler with a $90^{\circ} \mathrm{C}$ input temperature and it needs to be reduced to $55^{\circ} \mathrm{C}$ output temperature. A first cold current (F1) transports water that comes out from a decanter and it needs to be heated from 40 to $90^{\circ} \mathrm{C}$, while the second one (F2) transports a oil-hexane $(70 \%-30 \%)$ mixture with $60^{\circ} \mathrm{C}$ input and $90^{\circ} \mathrm{C}$ output temperatures. The Pinch Analysis was chosen as a synthesis methodology for the heat exchanger network synthesis. A cost analysis was carried out, verifying a 79,6\% steam consumption economy, after the pinch analysis-based energy integration, performing a 77,5\% (5.3\% overall consumption of plant) annual total cost reduction and showing a utilities investment return on three months.

Keywords: Pich Analysis, Energy Integration, Maximum Energy Recovery.

\footnotetext{
1 Programa de Pós-graduação Stricto Sensu em Engenharia Química da Universidade Estadual do Oeste do Paraná - UNIOESTE. Rua da Faculdade, 645. Jardim La Salle. CEP 85902-000. E-mail: f.espinoza@terra.com.br
} 


\section{INTRODUÇÃO}

A energia utilizada em processos de transformação está intrínseca em todas as considerações e análises feitas na elaboração e/ou otimização de uma planta industrial. O setor industrial volta grande parte de sua atenção à eficiência energética do processo, uma vez que ela é um importante componente do custo e, hoje em dia, além de se considerar o aspecto energético é preciso ainda levar em conta os aspectos ambientais. $\mathrm{O}$ calor não utilizado em um processo representa não somente um desperdício de combustível, mas também significa que será necessária maior quantidade de água de resfriamento e consequentemente mais poluição será gerada.

Dentro de qualquer indústria, aproveitar a energia térmica entre as correntes de processo, através de integração energética entre geradores e trocadores de calor e o sistema de arrefecimento é o caminho mais eficaz para se buscar a economia desejada. A síntese de rede de trocadores de calor (RTC) para integração energética de processos consiste em estabelecer uma determinada distribuição para um conjunto de trocadores, na qual o objetivo é obter a máxima recuperação energética (MRE) com um número mínimo de utilidades (NMU). Para a síntese de uma RTC podem ser utilizados métodos matemáticos ou métodos heurísticos (Análise Pinch).

Nos últimos anos surgiram diversos trabalhos com foco de aplicação industrial, nos mais variados setores. No processo de produção de papel e celulose (Sasazawa et al., 2000), em uma planta de óleo de soja (Maluf, 2003), em usinas de açúcar e álcool (Higa e Bannwart, 2003), no setor de energia (Pires, 2003), na etapa de extração por solvente de uma fábrica de produção de farelo branco de soja (Cereto, 2004), na área de refino e combustíveis (Moreira et al., 2007), na área petrolífera (Yoon e Park, 2007; Beninca, 2008; Rossi e Bannwart, 2009) e no refino e processamento de óleo (Paraíso, 2001).

Este trabalho apresenta um caso de aplicação de integração energética em uma planta de processamento de óleo vegetal, na etapa de extração do óleo. A metodologia adotada na síntese de RTC foi a Análise Pinch, através de um programa computacional em linguagem Fortran. Após a síntese de RTC foi realizada uma análise da viabilidade econômica, na qual a integração se mostrou financeiramente viável.

\section{METODOLOGIA}

A Análise Pinch foi a metodologia selecionada para alcançar a máxima (MRE) do processo, ou seja, encontrar a melhor forma de troca térmica entre as correntes quentes e frias aproveitando, assim, a energia do próprio processo para as operações de troca térmica.

Utilizando os conceitos da Análise Pinch foi desenvolvido um programa computacional em linguagem Fortran. O programa foi elaborado em forma modular, sendo composto por um programa principal e cinco módulos, conforme ilustrado na Figura 1.

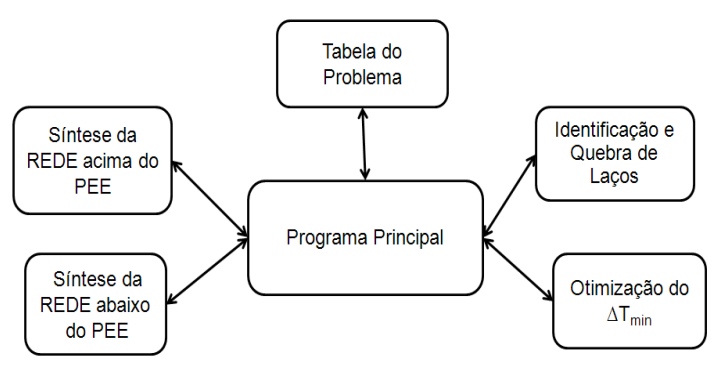

\section{Figura 1 - Diagrama de blocos para o programa desenvolvido}

O programa principal gerencia os demais módulos. As propriedades físicas devem ser fornecidas na temperatura média aritmética a qual é obtida a partir das temperaturas de entrada e de saída das correntes de processo. Os dados de entrada referentes às correntes são: número de correntes quentes e frias, temperatura de entrada e saída $\left({ }^{\circ} \mathrm{C}\right)$, produto entre vazão mássica e Capacidade Calorífica $(\mathrm{CP})\left(\mathrm{W} /{ }^{\circ} \mathrm{C}\right)$, coeficiente de transferência de calor convectivo $\left(\mathrm{W} / \mathrm{m}^{2}{ }^{\circ} \mathrm{C}\right)$ e o fator de incrustação $\left(\mathrm{m}^{2} \mathrm{C} / \mathrm{W}\right)$.

Primeiramente é acionado o módulo da otimização do $\Delta \mathrm{T}_{\text {mín }}$. Em seguida, é acionado o módulo Tabela do Problema, no qual determina-se o consumo mínimo de energia e divide-se o problema em duas regiōes, uma acima e outra abaixo do Ponto de Estrangulamente Energético (PEE). Feito isto, o programa principal irá acionar o módulo síntese da rede, onde será realizado o procedimento de síntese para as duas regiōes, obtendo-se assim a rede para a MRE, que poderá ser evoluída. Esta evolução é feita no módulo de identificação e quebra de laços. Os resultados são fornecidos em forma de tabelas, podendo ser 
impresso a Tabela do Problema e a rede evoluída contendo as temperaturas de entrada e saída, além do CP (produto entre vazão mássica e Capacidade Calorífica) da corrente quente e fria à qual pertence cada trocador.

\section{ESTUDO DE CASO}

A integração energética foi proposta para a etapa de extração de óleo de uma fábrica de óleo vegetal em operação na Região Oeste do Paraná, com produção média de 15000 ton/mês. Inicialmente foram avaliadas todas as correntes principais da etapa de extração, conforme apresentado na Figura 2. A Tabela 1 apresenta um resumo da avaliação realizada, onde são apresentadas as especificações das correntes que compóem a etapa de extração desta planta em operação.

Desta análise prévia, verificou-se a possibilidade de se realizar o processo de integração energética em quatro correntes (assinaladas em pontilhado vermelho na Figura 2), sendo duas correntes quentes e duas correntes frias. Duas dessas correntes fazem parte da separação da mistura líquida óleo e hexano, e as outras duas estão localizadas na parte final do processo de extração, na parte onde a água é aquecida até $90^{\circ} \mathrm{C}$ para evaporar os gases de hexano e depois segue para o sistema de tratamento de efluentes. As especificações das quatro correntes são apresentadas na Tabela 2 .

A primeira corrente quente denominada de Q1 (corrente 4, na Figura 2), consiste em uma corrente de óleo bruto que sai do evaporador 22 com vazão de 4,4 ton/h e temperatura de de entrada de $110^{\circ} \mathrm{C}$ e segue para o hidratador, sendo já preparada para a etapa seguinte que é o refino. A segunda corrente quente, denominada de corrente Q2 (corrente 17, na Figura 2), consiste em uma corrente de água com vazão de 4,0 ton/h que sai de um evaporador de solvente à temperatura de entrada de $90^{\circ} \mathrm{C}$ e saída de $55^{\circ} \mathrm{C}$. A corrente F1 (corrente 16, na Figura 2) consiste em uma corrente de água que sai do decantador a uma vazão de 4,0 ton/h e a uma temperatura de $40^{\circ} \mathrm{C}$, que recebe o fluxo da coluna $60 S 1$ e do condensador 19. A corrente F2 (corrente 2, na Figura 2) consiste em uma corrente de miscela (mistura 70 $\%$ óleo e $30 \%$ hexano) que segue para a coluna $18 \mathrm{~S}$ e tem uma vazão de 5,5 ton/h com temperatura de entrada de $60^{\circ} \mathrm{C}$ e temperatura de saída de $90^{\circ} \mathrm{C}$.
A Corrente Q1 vai para a etapa seguinte, hidratação, à temperatura de $110^{\circ} \mathrm{C}$. Necessita ser resfriada e seguir para a degomagem a menos de $90^{\circ} \mathrm{C}$, pois neste processo utiliza-se a degomagem com água (hidratação), que é favorecida a temperaturas abaixo de $100{ }^{\circ} \mathrm{C}$. Neste caso uma menor temperatura (entre 70 e $80^{\circ} \mathrm{C}$ ) irá produzir uma melhor degomagem por facilitar a hidratação dos fosfatídeos. A corrente Q2 consiste na água que sai do fervedor (17), que deve ser aquecida até $90^{\circ} \mathrm{C}$. Esta corrente é direcionada para a estação de tratamento de efluentes e não deve chegar ao efluente nesta temperatura, sendo ideal ser resfriada pelo menos até $55^{\circ} \mathrm{C}$. Tendo em vista que é uma vazão de 4 ton/h, se esta corrente pudesse trocar calor com uma corrente fria teria um potencial de fornecer $163 \mathrm{~kW} / \mathrm{h}$.

A corrente $\mathrm{F} 1$, que consiste de água oriunda do decantador, recebe o fluxo dos condensadores 19 e 23 , e necessita ser aquecida até $90^{\circ} \mathrm{C}$ para eliminação dos gases residuais de hexano. $\mathrm{O}$ aquecimento desta corrente que tem vazão de 4,0 ton/h e consome $232,5 \mathrm{~kW} / \mathrm{h}$, energia esta provida atualmente com utilidade quente. A corrente F2, que consiste na miscela, entra na coluna $18 \mathrm{~S}$ e necessita ser aquecida a $90^{\circ} \mathrm{C}$ e seu aquecimento consome $98 \mathrm{~kW} / \mathrm{h}$, energia esta também fornecida atualmente com utilidade quente. As duas correntes frias F1 e F2, geram um gasto de energia de $330,6 \mathrm{~kW} / \mathrm{h}$, levando-se em consideração que a fábrica de óleo trabalha em geral em regime de 24 horas/dia, 335 dias por ano, este consumo atinge um valor de $2658 \mathrm{MW} /$ ano aproximadamente.

Após definição das correntes e suas necessidades energéticas, antes da síntese da RTC, foi realizado a otimização do $\Delta \mathrm{T}_{\min }$ utilizando o procedimento proposto por Ahmad e Linnhoff (1985). O cálculo foi feito estabelecendo-se metas para o consumo de utilidades, número de unidades, área de transferência de calor e custo total anual. O procedimento de calculo consiste em encontrar a MRE e calcular a área mínima de troca térmica para a rede a ser sintetizada. Este cálculo é baseado no diagrama temperatura $\mathrm{x}$ entalpia (TH), apresentado por Tjoe e Linnhoff (1984), onde os intervalos de entalpia no diagrama $\mathrm{TH}$ são obtidos para cada mudança de inclinação nas curvas compostas. Assim, aplicando o escoamento em contracorrente às curvas compostas, Tjoe e Linnhoff (1984) chegaram a seguinte expressão para à área mínima. 


$$
A_{\text {MIN }}=\sum_{i=1}^{n} \frac{1}{F_{i} \cdot \operatorname{MLDT}_{i}} \cdot\left(\sum_{j=1}^{m} \frac{q_{j}}{h_{j}}\right)_{i}
$$

Sendo, $\mathrm{n}=$ número de intervalos; $\mathrm{m}=$ número de correntes; $\mathrm{q}_{\mathrm{j}}=$ energia transferida pelas correntes $j$ (quentes e frias) existentes no intervalo $\mathrm{i} ; \mathrm{h}_{\mathrm{i}}=$ coeficiente de transferência de calor convectivo (incluindo incrustação) das correntes $\mathrm{j}$, também presentes no intervalo $\mathrm{i} ; \mathrm{MLDT}_{\mathrm{i}}=$ média logarítmica da diferença de temperatura para cada intervalo, calculada para o escoamento em contracorrente; $\mathrm{FT}_{\mathrm{i}}=$ fator de correção para o MLDT do intervalo i.

O custo de capital de uma RTC é função da área de transferência de calor e do número de equipamentos de troca térmica. Desta forma, uma boa estimativa para o custo de capital depende da maneira como estas variáveis estão relacionadas. Geralmente, o custo de capital está relacionado aos trocadores da seguinte forma:

$$
C E Q=a+b \cdot A^{C}
$$

Sendo $A$ é a área de troca térmica e $a, b$ e $c$ são constantes, sendo que $0<c \leq 1$.

O custo total da rede, relativo aos trocadores, será a soma dos custos de cada trocador. No entanto, na fase de determinação do $\Delta \mathrm{T}_{\min }$ ótimo a rede ainda não é conhecida e o custo deverá ser estimado em função da área mínima e do número de unidades. Segundo Ahmad e Linnhoff (1985), o custo relativo ao capital pode ser dado por:

$$
\mathrm{CEQ}=\mathrm{U}_{\mathrm{MIN}} \cdot\left[\mathrm{a}+\mathrm{b} \cdot\left(\frac{\mathrm{A}_{\mathrm{MIN}}}{\mathrm{U}_{\mathrm{MIN}}}\right)^{\mathrm{C}}\right]
$$

Sendo $\mathrm{U}_{\mathrm{MIN}}=$ número mínimo de unidades; $\mathrm{A}_{\mathrm{MIN}}=$ área mínima de transferência de calor; $\mathrm{a}, \mathrm{b}, \mathrm{c}=$ constantes.

O custo anual relativo ao capital é calculado utilizando-se uma taxa de atratividade i e assumindo-se um tempo de vida útil para os equipamentos $\mathrm{T}$, no qual o capital será depreciado. Assim:

$$
\mathrm{CEQ}_{\mathrm{ANUAL}}=\mathrm{CEQ} \cdot \frac{1}{\mathrm{~T}} \cdot(1+\mathrm{i})^{\mathrm{T}}
$$

O custo relativo às utilidades é calculado em função dos custos e consumos mínimo das utilidades quente e fria.
CENER $=$ HOP $\cdot\left(\sum_{\mathrm{i}=1}^{\mathrm{n}}(\mathrm{UTQ} \cdot \mathrm{CQ})+\sum_{\mathrm{i}=1}^{\mathrm{m}}(\mathrm{UTF} \cdot \mathrm{CF})\right)$

Sendo HOP $=$ horas de operação da planta por ano; UTQ = consumo da utilidade quente $\mathrm{kW}$; UTF = consumo da utilidade fria $\mathrm{kW}$; CQ = custo da utilidade quente (dólares $/ \mathrm{kW}) ; \mathrm{CF}=$ custo da utilidade fria (dólares/kW).

Assim, o custo total anual é dado por:

$\mathrm{CT}_{\mathrm{ANUAL}}=\mathrm{CEQ}_{\mathrm{ANUAL}}+\mathrm{CENER}$

Utilizando-se o programa computacional, foi feita a iteração para se achar o $\Delta \mathrm{T}_{\text {mín }}$ ótimo, e trabalhou-se na faixa de 5-30 ${ }^{\circ} \mathrm{C}$, variando-se a temperatura de 5 em $5^{\circ} \mathrm{C}$. A temperatura na qual se obteve o menor custo foi a de valor $10^{\circ} \mathrm{C}$; portanto, o $\Delta \mathrm{T}_{\text {mín }}$ ótimo para essas quatro correntes em análise é de $10^{\circ} \mathrm{C}$, como se observa na Figura 3.

Os dados utilizados de custos com utilidades foram característicos da região do Paraná. Os valores das variáveis utilizadas para o cálculo dos custos são apresentados na Tabela 3 .

Após o cálculo do $\Delta \mathrm{T}_{\min }$ ótimo, o próximo passo foi a construção da Tabela do Programa, apresentada na Tabela 4, e da cascata energética para este mesmo $\Delta \mathrm{T}_{\text {mín }}$, apresentada na Figura 4.

Avaliando a Tabela 4, observa-se que há a necessidade de $91 \mathrm{~kW}$ de utilidades quentes para suprir a necessidade de aquecimento das correntes frias. Ocorreu neste caso, com $\Delta \mathrm{T}_{\text {mín }}$ ótimo de 10 ${ }^{\circ} \mathrm{C}$, que toda a troca térmica entre correntes ocorre apenas na zona acima do PEE.

Em seguida fez-se a análise, utilizando o programa computacional, das possibilidades de integração e alocação de trocadores nas correntes. Notou-se que as correntes F1 e F2 têm necessidades entálpicas de $232,5 \mathrm{~kW}$ e $98,1 \mathrm{~kW}$, respectivamente. A corrente Q1 tem 78,1 kW para trocar enquanto a corrente Q2 tem 168,2 kW. Como a corrente Q2 tem a restrição de não poder trocar calor com a corrente F2, então, a combinação deve ser feita com a corrente F1, podendo assim ceder todo o seu calor $(168,2 \mathrm{~kW})$. A corrente Q1 trocaria calor então, com a corrente F2, cedendo $78,1 \mathrm{~kW}$. Ao fim da análise optou-se pela utilização de dois trocadores de calor e as necessidades remanescentes de aquecimento seriam supridas por utilidades quentes, que são equipamentos já existentes na linha. Pela Figura 4 observa-se que não haverá consumo de utilidades frias. A temperatura de saída da corrente Q2 pode ser reduzida 
de 55 para $50{ }^{\circ} \mathrm{C}$. Assim o calor trocado no trocador 2 aumentaria de $162,8 \mathrm{~kW}$ para $186,0 \mathrm{~kW}$ e a corrente F1 teria uma temperatura de saída de $80^{\circ} \mathrm{C}$, e ainda reduz-se a quantidade de utilidade quente para aquecer a corrente $\mathrm{F} 1 \mathrm{de} 69,8 \mathrm{~kW}$ para $46,6 \mathrm{~kW}$. A Figura 5 apresenta o diagrama de grade da rede proposta pela, para o $\Delta \mathrm{T}_{\text {mín }}$ de $10{ }^{\circ} \mathrm{C}$.

O trocador 1 vai integrar a corrente Q1 com a corrente F2, trocando $76,8 \mathrm{~kW}$, energia que é atualmente suprida por utilidades quentes. Pelo cálculo projetado, este trocador terá uma área de troca térmica de $7,0 \mathrm{~m}^{2}$, segundo empresa especializada em fabricação de equipamentos deste porte. A corrente Q1 atingirá sua temperatura final de $80^{\circ} \mathrm{C}$, enquanto que a corrente F2 atingirá a temperatura final de $83,5^{\circ} \mathrm{C}$, sendo necessário ainda consumir $21,3 \mathrm{~kW}$ através de utilidades quentes para se atingir a temperatura final desejada de $90^{\circ} \mathrm{C}$. O trocador 2 vai integrar a corrente Q2 de com a corrente F1, trocando $186 \mathrm{~kW}$, energia que também é suprida por utilidades quentes. A área de troca térmica deste trocador será de $8,2 \mathrm{~m}^{2}$, também segundo empresa especializada em fabricação de equipamentos. A corrente Q2 atingirá a temperatura final de $50^{\circ} \mathrm{C}$, temperatura razoável para ser enviado para a estação de tratamento de efluentes. Já a corrente F1 atingirá a temperatura de $80^{\circ} \mathrm{C}$, sendo necessário o consumo de 46,6 $\mathrm{kW}$, através de utilidade quente para se atingir a temperatura final de $90{ }^{\circ} \mathrm{C}$. Observa-se que no sistema não haverá consumo de utilidade fria.

$\mathrm{O}$ custo total dos 2 trocadores será de $\mathrm{R} \$$ 16460,00 , segundo orçamento realizado por uma empresa especializada em fabricação de equipamentos. O trocador de calor é do tipo placas paralelas de aço inox.

A Figura 6 representa a rede proposta, com dois trocadores de calor integrados à planta. $\mathrm{Na}$ Figura 6(a) está evidenciada a troca de calor realizada pelo trocador 1, entre as correntes Q1 e F2, e na Figura 6(b) evidencia-se a troca de calor realizada pelo trocador 2, entre as correntes Q2 e F1.

O comparativo entre os resultados antes e após a integração energética. São apresentados na Tabela 5. A economia de consumo de vapor obtida é de 79,6\% em relação ao modelo sem a integração energética, o que significa uma redução de $5,3 \%$ de redução do consumo global de vapor da planta, gerando uma economia de $\mathrm{R} \$$ 91120,00/ ano em vapor. A redução de custo total obtida na integração energética proposta é de $\mathrm{R} \$ 88655,60$ / ano, que equivale a $77,5 \%$ de redução em relação ao custo, sem a integração, considerando-se uma planta que opera $24 \mathrm{~h} / \mathrm{dia}, 335 \mathrm{dias} / \mathrm{ano}$, com eficiência operacional de $95 \%$. O custo anual de capital é de $\mathrm{R} \$ 2465,00$. A taxa de retorno para o investimento proposto é de apenas 3 meses.

\section{CONCLUSÕES}

Neste trabalho foi realizado um estudo de caso da otimização energética, utilizando a Análise Pinch, da etapa de extração de uma fábrica de óleo de soja em operação, com produção média de 15000 ton/mês. Foi constatada, após a integração energética, uma redução no custo com utilidades anual de 79,6\% e no custo total anual de $77,5 \%$.

Verificou-se que o custo de vapor tem relação direta com o tipo de matéria-prima usada na caldeira. No caso estudado foi utilizado madeira, cujo cultivo e renovação é complexo e demorado. Assim, verifica-se um duplo benefício com a integração energética: o econômico e o ambiental.

Desta forma, comprovou-se a eficácia do programa computacional e também da Análise Pinch na integração energética de processos quando aplicadas à plantas em operação, pois além da aplicação simples e da forte interação com o engenheiro de processo é possível obter resultados cuja economia de energia térmica seja muito significativa, contribuindo desta forma para processos industriais mais eficientes.

\section{NOMENCLATURA}

\begin{tabular}{|l|l|}
\hline a & Constante; \\
\hline $\mathrm{A}_{\text {mín }}$ & $\begin{array}{l}\text { Área mínima de transferência de calor } \\
\left(\mathrm{m}^{2}\right) ;\end{array}$ \\
\hline $\mathrm{b}$ & Constante; \\
\hline $\mathrm{c}$ & Constante; \\
\hline $\mathrm{C}_{\text {capital }}$ & Custo capital (dólares); \\
\hline $\mathrm{C}_{\text {energ }}$ & Custo energético (dólares $/ \mathrm{kW}) ;$ \\
\hline $\mathrm{C}_{\mathrm{EQ}}$ & Custo com equipamentos; \\
\hline $\mathrm{C}_{\mathrm{EQ} \text { anual }}$ & Custo com equipamentos anual; \\
\hline $\mathrm{C}_{\mathrm{F}}$ & Custo da utilidade fria $($ dólares $/ \mathrm{kW}) ;$ \\
\hline $\mathrm{C}_{\text {global }}$ & Custo global (dólares); \\
\hline $\mathrm{CP}$ & Capacidade calorífica $\left(\mathrm{kW} / \mathrm{o}^{\mathrm{C}}\right) ;$ \\
\hline
\end{tabular}




\begin{tabular}{|c|c|}
\hline $\mathrm{CPF}$ & $\begin{array}{l}\text { Capacidade térmica das correntes frias ou } \\
\text { correntes ramificadas }\left(\mathrm{kW} /{ }^{\circ} \mathrm{C}\right) \text {; }\end{array}$ \\
\hline CPQ & $\begin{array}{l}\text { Capacidade térmica das correntes quentes } \\
\text { ou correntes ramificadas }\left(\mathrm{kW} / \mathrm{o}^{\circ} \mathrm{C}\right)\end{array}$ \\
\hline $\mathrm{C}_{\mathrm{Q}}$ & Custo da utilidade quente (dólares/kW); \\
\hline $\mathrm{C}_{\text {util. }}$ & Custo com utilidades; \\
\hline $\mathrm{H}_{\mathrm{OP}}$ & Horas de operação da planta por ano; \\
\hline i & Taxa de atratividade; \\
\hline $\mathrm{L}$ & Número de laços (loops); \\
\hline M & Vazão mássica da corrente $(\mathrm{kg} / \mathrm{h})$; \\
\hline MRE & Máxima recuperação energética $(\mathrm{kW})$; \\
\hline NMU & Número mínimo de utilidades; \\
\hline PEE & Ponto de estrangulamento energético; \\
\hline RTC & Rede de trocadores de calor; \\
\hline SR & Sub-rede; \\
\hline $\mathrm{T}$ & Temperatura $\left({ }^{\circ} \mathrm{C}\right)$ \\
\hline $\mathrm{t}$ & $\begin{array}{l}\text { Tempo de vida útil dos trocadores de calor } \\
\text { (anos); }\end{array}$ \\
\hline $\mathrm{U}$ & $\begin{array}{l}\text { Número de equipamentos de troca } \\
\text { térmica; }\end{array}$ \\
\hline $\mathrm{U}_{\min }$ & $\begin{array}{l}\text { Número mínimo de unidades trocadoras } \\
\text { de calor; }\end{array}$ \\
\hline $\mathrm{U}_{\text {mínMRE }}$ & $\begin{array}{l}\text { Número mínimo de unidades trocadoras } \\
\text { de calor para uma máxima recuperação } \\
\text { energética; }\end{array}$ \\
\hline $\mathrm{UT}_{\mathrm{F}}$ & Consumo da utilidade fria $(\mathrm{kW})$ \\
\hline $\mathrm{UT}_{\mathrm{Q}}$ & Consumo da utilidade quente $(\mathrm{kW})$; \\
\hline$\Delta \mathrm{H}$ & Variação de entalpia $(\mathrm{kW})$ \\
\hline$\Delta \mathrm{T}$ & Variação de temperatura $\left({ }^{\circ} \mathrm{C}\right)$ \\
\hline$\Delta \mathrm{T}_{1}$ & $\begin{array}{l}\text { Diferença de temperatura em um extremo } \\
\text { do intervalo }\left({ }^{\circ} \mathrm{C}\right) ;\end{array}$ \\
\hline$\Delta \mathrm{T}_{2}$ & $\begin{array}{l}\text { Diferença de temperatura no outro } \\
\text { extremo do intervalo }\left({ }^{\circ} \mathrm{C}\right)\end{array}$ \\
\hline$\Delta \mathrm{T}_{\text {mín }}$ & $\begin{array}{l}\text { Diferença de temperatura mínima entre } \\
\text { os terminais do trocador }\left({ }^{\circ} \mathrm{C}\right) \text {. }\end{array}$ \\
\hline
\end{tabular}

\section{REFERÊNCIAS}

AHMAD, S. LINNHOFF, B. (1985). Overall cost targets for heat Exchange networks. ICHemE Annual Res. Meeting, p. $51-56$.

BENINCA, M. (2008). Integração energética de uma planta de eteno com aspectos de flexibilidade: Aplicação da Análise Pinch em conjunto com a otimização matemática. Dissertação Mestrado apresentada na Faculdade de Engenharia Química da Universidade Federal do Rio grande do Sul.
CERETO, A.C. (2004). Integração da Rede de Trocadores de Calor em Extração por Solvente para a Produção de Farelo Branco de Soja. Dissertação de Mestrado apresentada na Faculdade de Engenharia Química da Universidade Federal do Rio Grande do Sul.

HIGA, M. BANNWART, A.C. (2003). Avaliação energética em usina de açúcar e álcool utilizando a Análise Pinch. Revista Iberoamericana de Ingeniería Mecánica. Vol. 9 (3), p. 95-101.

MALUF, J.U. (2003). Integração energética da etapa de extração de óleo de soja da Cocamar. Dissertação de Mestrado apresentada na Faculdade de Engenharia Química da Universidade Estadual de Maringá.

MOREIRA, A. M. PELLANDA, A. P. VELOZ, C.S.C. BRUTIMAVICIUS, P. J.;PORTO, M. F.;AHÓN, V. R. R.;COSTA, A. L. H. (2007). Estudo da integração energética de uma unidade de destilação atmosférica e a vácuo. 4을 PDPETRO 4.2.0198.4-1, Campinas.

PARAÍSO, P.R. (2001). Modelagem e análise do processo de obtenção do óleo de soja. Tese de Doutorado apresentada na Faculdade de Engenharia Química da Universidade Estadual de Campinas.

PIRES, V.F.M. (2003). Análise energética global de um processo petroquímico. Dissertação de Mestrado apresentada na Faculdade de Engenharia Química na - Universidade Estadual de Campinas, Campinas.

ROSSI, L.F.S.; BANNWART, A.C. (2009). Otimização da etapa de pré-aquecimento de uma carga de uma unidade de craqueamento catalítico, fazendo uso da programação matemática e da Metodologia Pinch na recuperação energética de redes. Pesquisa Operacional para o Desenvolvimento. Vol.1 (1), p.21-32.

SASAZAWA, R.S.; RAVAGNANI, M. A. S. S.; PESCH, L. R. (2000). Integração energética no processo de papel e celulose. Congreso Ibero americano de investigacion em celulosa y papel. 
TJOE, T. N. LINNHOFF, B. (1984). Using Pinch Technology for Process Retrofit. Chemical Engineering, Vol. 93, p. 47-60.
YOON, S.G.; LEE, J.; PARK, S. (2007) Heat integration analysis for an industrial ethilbenzene plant using Pinch Analysis. Applied Thermal Engineering. Vol. (27), p. 886-893.

\section{ANEXO - Tabelas e Figuras}

Tabela 1 - Especificaçõos das correntes da etapa de extração

\begin{tabular}{|c|c|c|c|c|c|}
\hline Corrente & Descrição & $\begin{array}{c}\text { Vazão } \\
(\mathrm{t} / \mathrm{h})\end{array}$ & $\begin{array}{l}\mathrm{T}_{\text {ent }} \\
\left({ }^{\circ} \mathrm{C}\right)\end{array}$ & $\begin{array}{l}\mathrm{T}_{\text {saída }} \\
\left({ }^{\circ} \mathrm{C}\right)\end{array}$ & $\begin{array}{c}\mathrm{Cp} \\
\left(\mathrm{kW} / \mathrm{kg}^{\circ} \mathrm{C}\right)\end{array}$ \\
\hline 0 & Massa expandida & 22 & 60 & 60 & 0,60 \\
\hline 1 & Miscela entrada 60 & 22 & 60 & 60 & 0,51 \\
\hline 2 & Miscela entrada 18 & 5,5 & 60 & 90 & 0,51 \\
\hline 3 & Miscela entrada 22 & 4,5 & 90 & 110 & 0,51 \\
\hline 4 & Óleo bruto hidratado & 4,4 & 110 & 80 & 0,52 \\
\hline 5 & Óleo bruto hidratado & 4,4 & 80 & 80 & 0,52 \\
\hline 10 & Óleo degomado para armazenagem & 4,4 & 80 & 35 & 0,56 \\
\hline 11 & Gases de hexano & 17,5 & 80 & 45 & 0,53 \\
\hline 12 & Gases de hexano & 0,1 & 80 & 45 & 0,53 \\
\hline 13 & Vapor & 4 & $120-180$ & 90 & --- \\
\hline 14 & Farelo tostado & 16,3 & $30-40$ & $30-40$ & --- \\
\hline 15 & Água + hexano & 18,6 & 30 & 40 & 0,70 \\
\hline 16 & Água decantada & 4 & 40 & 90 & 1,0 \\
\hline 17 & Água saída do fervedor & 4 & 90 & 40 & 1,0 \\
\hline 18 & Óleo absorção & 2 & 16 & 20 & 0,50 \\
\hline 19 & Óleo desorção & 2 & 90 & 88 & 0,50 \\
\hline 20 & Hexano líquido + água & 3 & 50 & 50 & 0,70 \\
\hline 21 & Gases de hexano + vapor & 8 & 90 & 80 & 0,53 \\
\hline 22 & Condensado hexano + vapor & 7 & 60 & 60 & 0,70 \\
\hline 23 & Gases de hexano + vapor & 1 & 80 & 70 & 0,53 \\
\hline 24 & Hexano líquido & 28,6 & 50 & 50 & 0,53 \\
\hline 25 & Utilidade água & 400 & 24 & 26 & 1,0 \\
\hline 26 & Utilidade água & 400 & 26 & 28 & 1,0 \\
\hline 27 & Utilidade água & 400 & 28 & 30 & 1,0 \\
\hline 28 & Utilidade água & 400 & 30 & 32 & 1,0 \\
\hline 29 & Hexano gás & 0,2 & 40 & 80 & 0,54 \\
\hline 30 & Vapor indireto (até $4 \mathrm{kgf} / \mathrm{cm}^{2}$ ) & 0,24 & 145 & 120 & \\
\hline 31 & Vapor direto $\left(7 \mathrm{kgf} / \mathrm{cm}^{2}\right)$ & 0,082 & 170 & 160 & ---- \\
\hline 32 & Hexano gás & 0,2 & 90 & 90 & 0,53 \\
\hline 33 & Vapor & 0,24 & 170 & 160 & --- \\
\hline
\end{tabular}




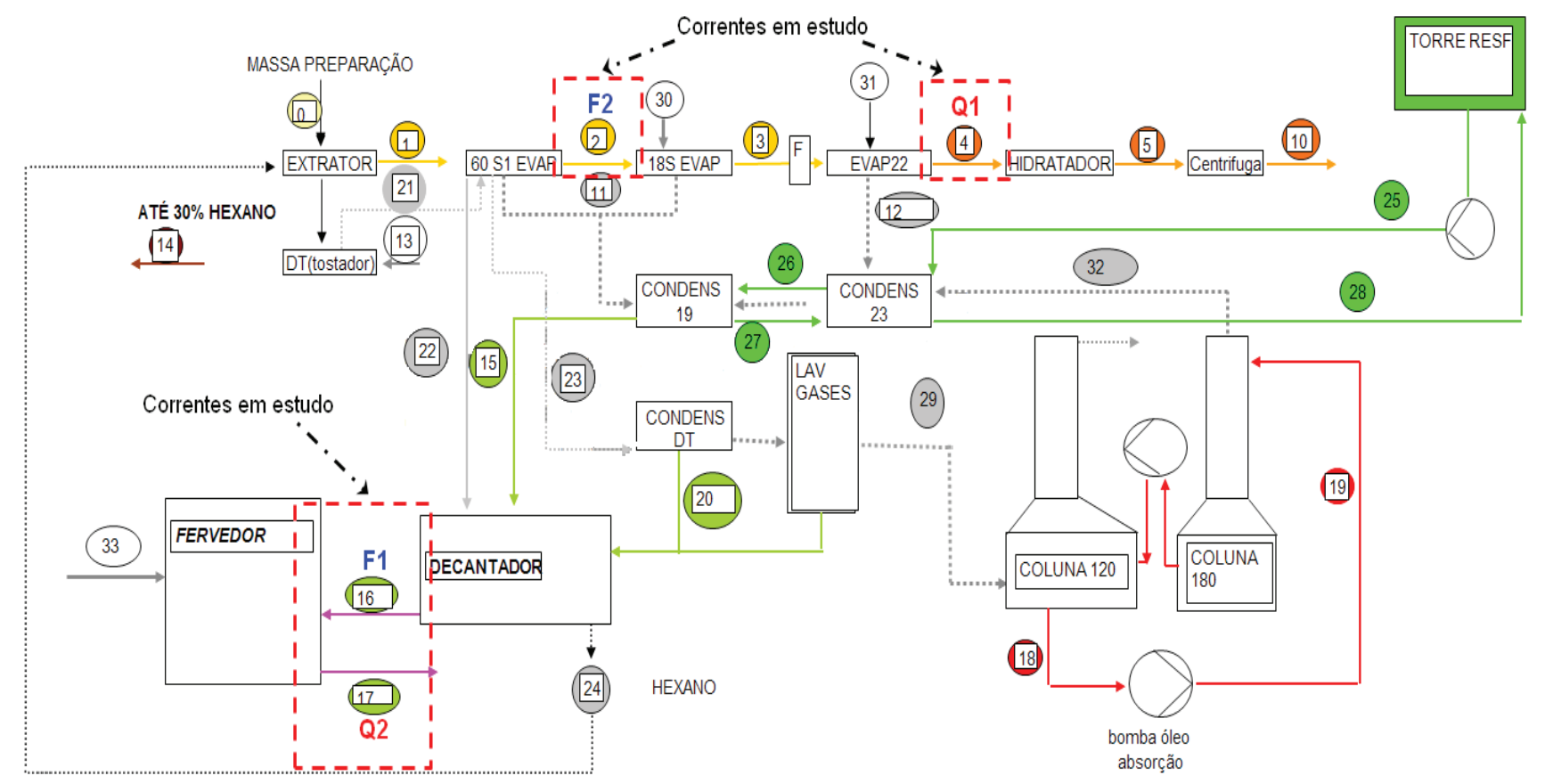

Figura 2 - Representação gráfica da etapa de extração da planta de óleo de soja

Tabela 2 - Correntes priorizadas no estudo e suas especificações

\begin{tabular}{|c|c|c|c|c|c|}
\hline Corrente & $\begin{array}{c}\text { Vazão mássica } \\
(\mathrm{t} / \mathrm{h})\end{array}$ & $\begin{array}{c}\mathrm{MCp^{* }}(\mathrm{kW} / \\
\left.{ }^{\circ} \mathrm{C}\right)\end{array}$ & $\mathrm{T}_{\text {Entrada }}\left({ }^{\circ} \mathrm{C}\right)$ & $\mathrm{T}_{\text {Sáda }}\left({ }^{\circ} \mathrm{C}\right)$ & $\mathrm{Q}(\mathrm{kW})$ \\
\hline Q1- Quente - óleo bruto hidratador (4) & 4,4 & 2,56 & 110 & 80 & $-76,8$ \\
\hline Q2- Quente - água saída fervedor (17) & 4,0 & 4,65 & 90 & 55 & $-162,8$ \\
\hline F1- Fria - água decantada (16) & 4,0 & 4,65 & 40 & 90 & 232,5 \\
\hline F2- Fria - miscela entrada (2) & 5,5 & 3,27 & 60 & 90 & 98,1 \\
\hline *vazão mássica x Capacidade Calorífica & & & & & \\
\hline
\end{tabular}

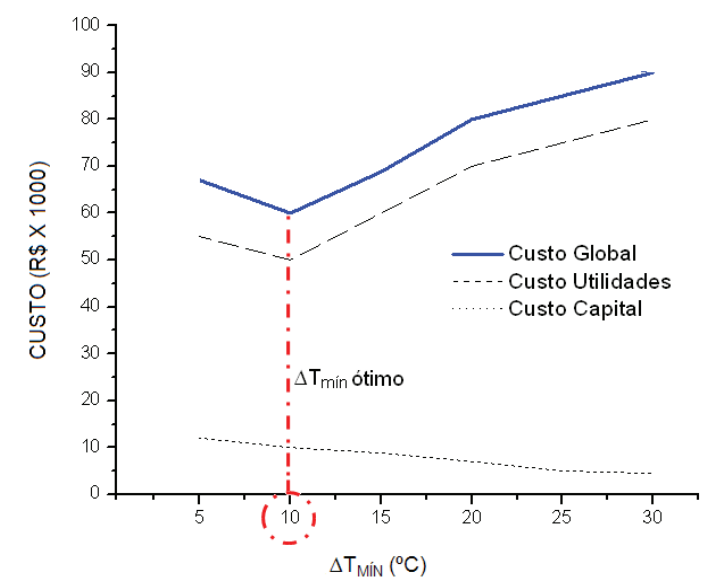

Figura 3 - Variação do custo com $\Delta \mathrm{T}_{\text {mín }}$ ótimo 
Tabela 3 - Valores das variáveis utilizadas para os cálculos

\begin{tabular}{|c|c|}
\hline Variável & Valor Utilizado \\
\hline $\mathrm{H}_{\mathrm{op}}$ & $7638(\mathrm{~h} / \mathrm{ano})$ \\
\hline $\mathrm{UT}_{\mathrm{Q}}$ & $1.830 .064,8(\mathrm{~kW})$ \\
\hline $\mathrm{C}_{\mathrm{Q}}$ & $0,02(\mathrm{R} \$ / \mathrm{kW} . \mathrm{h})$ \\
\hline $\mathrm{UT}_{\mathrm{F}}$ & $2.521 .303,8(\mathrm{~kW})$ \\
\hline $\mathrm{C}_{\mathrm{F}}$ & $0,0015(\mathrm{R} \$ / \mathrm{kW} \cdot \mathrm{h})$ \\
\hline $\mathrm{a}$ & $4816,5(\mathrm{R} \$)$ \\
\hline $\mathrm{b}$ & $2755,1\left(\mathrm{R} \$ / \mathrm{m}^{2}\right)$ \\
\hline $\mathrm{c}$ & 0,8 \\
\hline $\mathrm{A}$ & $15,2\left(\mathrm{~m}^{2}\right)$ \\
\hline $\mathrm{i}$ & 0,08 \\
\hline $\mathrm{t}$ & $10(\mathrm{anos})$ \\
\hline
\end{tabular}

Tabela 4 - Tabela do problema para o caso estudado, utilizando $\Delta \mathrm{T}_{\text {mín }}$ de $10{ }^{\circ} \mathrm{C}$

\begin{tabular}{|c|c|c|c|c|c|c|c|c|c|}
\hline & \multicolumn{2}{|c|}{$\begin{array}{c}\text { Corrente Fria } \\
\left({ }^{\circ} \mathrm{C}\right)\end{array}$} & \multicolumn{2}{|c|}{$\begin{array}{c}\text { Corrente Quente } \\
\qquad\left({ }^{\circ} \mathrm{C}\right)\end{array}$} & \multirow[t]{2}{*}{$\begin{array}{l}\text { Déficit } \\
(\mathrm{kW})\end{array}$} & \multicolumn{2}{|c|}{$\begin{array}{l}\text { Acúmulo } \\
\text { (kW) }\end{array}$} & \multicolumn{2}{|c|}{$\begin{array}{l}\text { Fluxo de calor } \\
\qquad(\mathrm{kW})\end{array}$} \\
\hline & $\mathrm{F} 1$ & F2 & Q1 & Q2 & & Entrada & Saída & Entrada & Saída \\
\hline & 100 & & 110 & & & & & & \\
\hline SR1 & 90 & & 100 & & $-25,6$ & 0 & 25,6 & 91 & 116,5 \\
\hline SR2 & 80 & 4 & 90 & ? & 53,6 & 25,6 & -28 & 116,5 & 63 \\
\hline SR3 & 70 & & 80 & & 7,1 & -28 & $-35,1$ & 63 & 55,9 \\
\hline SR4 & 60 & 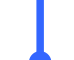 & 70 & & 32,7 & $-35,1$ & $-67,8$ & 55,9 & 23,3 \\
\hline SR5 & 45 & & 55 & 7 & 0 & $-67,8$ & $-67,8$ & 23,3 & 23,3 \\
\hline SR6 & 40 & & 50 & & 23,3 & $-67,8$ & -91 & 23,3 & 0 \\
\hline
\end{tabular}




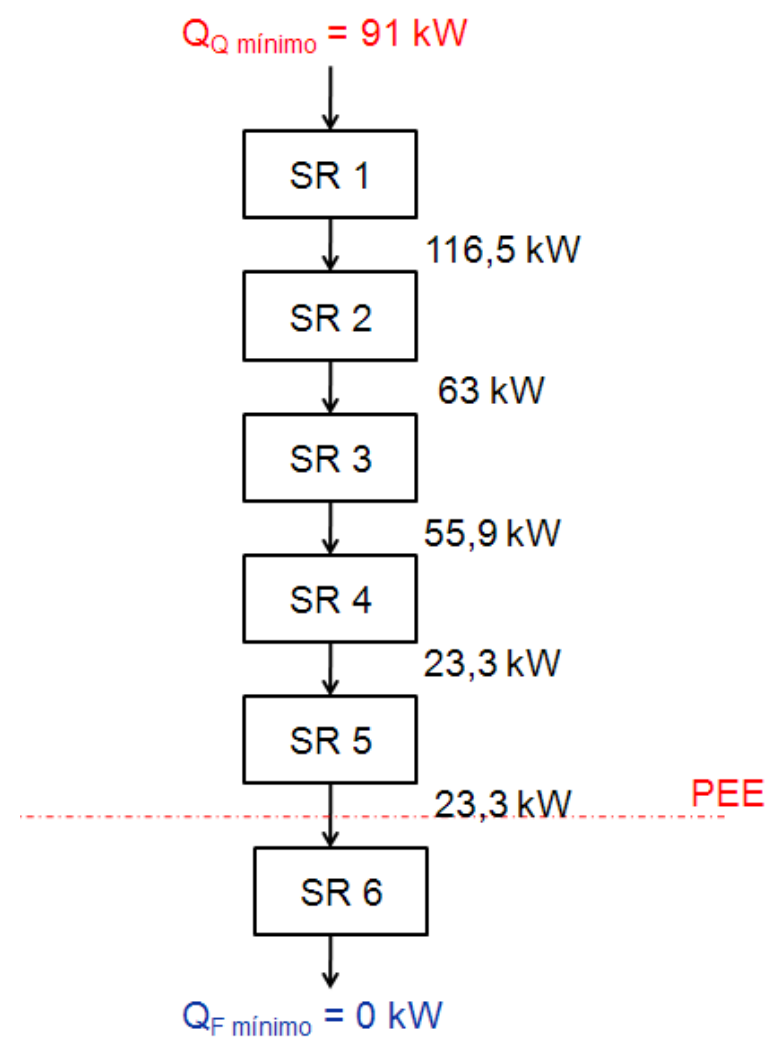

Figura 4 - Cascata energética para $\Delta$ Tmín de $10^{\circ} \mathrm{C}$

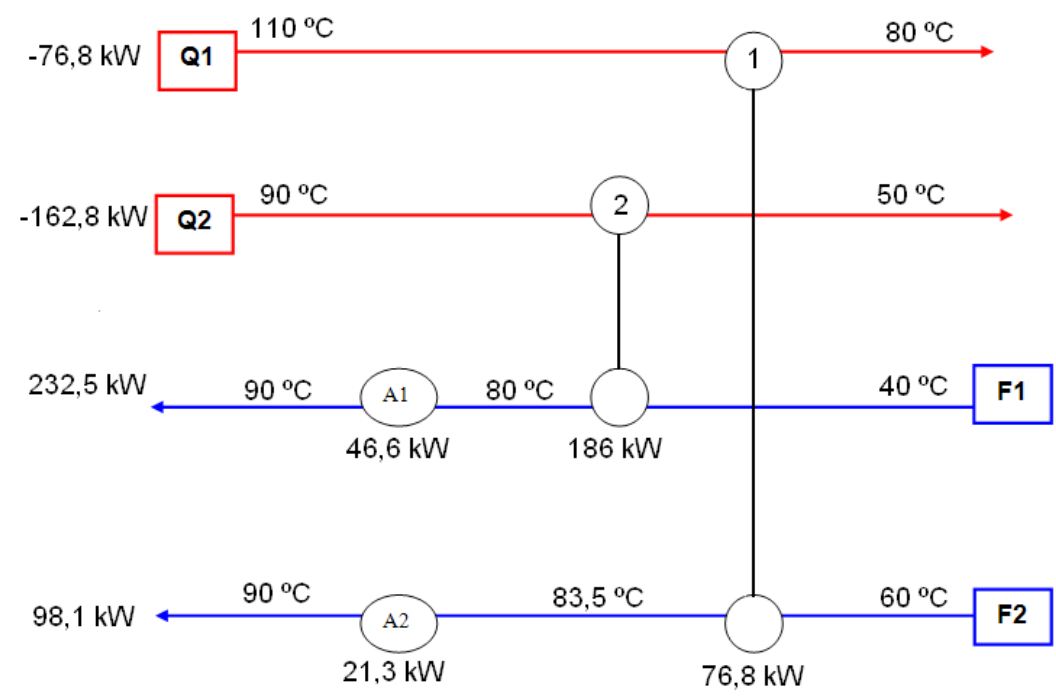

Figura 5 - Diagrama de grade da rede proposta para $\Delta$ Tmín de $10{ }^{\circ} \mathrm{C}$, reduzindo a temperatura da corrente $\mathrm{Q} 2$ de $55^{\circ} \mathrm{C}$ para $50^{\circ} \mathrm{C}$ 


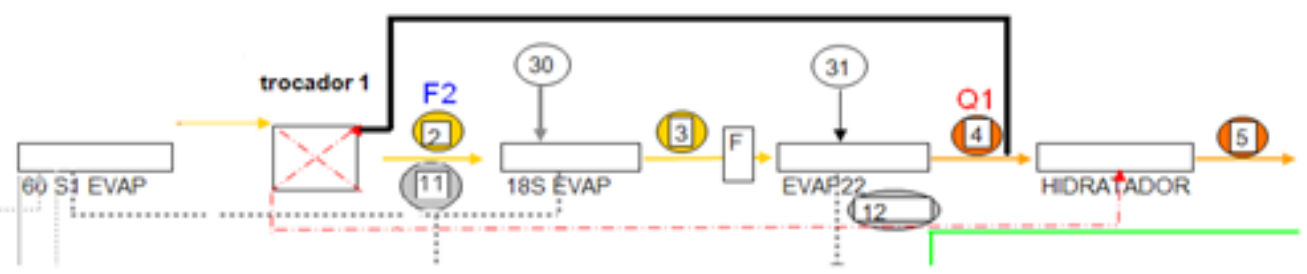

(a)

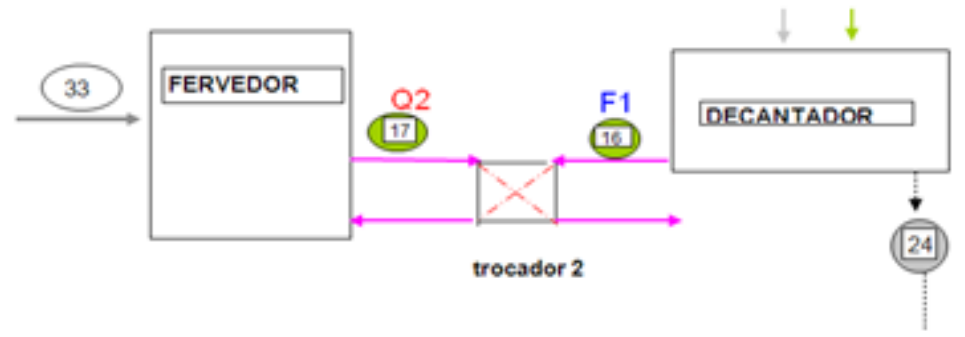

(b)

Figura 6 - Representação gráfica das correntes de processo da etapa de extração da planta de óleo de soja, com dois trocadores integrados

Tabela 5 - Comparativo custo antes e depois da proposta de otimização da rede

\begin{tabular}{|c|c|c|c|}
\hline & Custo utilidades anual (R\$) & Custo de capital anual (R\$) & Custo total anual (R\$) \\
\hline Antes da integração & $114.455,6$ & ---- & $114.455,6$ \\
\hline Após a integração & $23.334,9$ & $2.465,0$ & $25.799,9$ \\
\hline Redução de custo & $91.120,6$ & ---- & $88.655,6$ \\
\hline \% total de redução & 79,6 & ---- & 77,5 \\
\hline
\end{tabular}

